Inhaltlich orientieren sich die Prinzipien im Wesentlichen an denselben Standards, die innerhalb der Sicherheitsindustrie entwickelt wurden.

Bei der Sensibilisierung der Firmen und ihrer Mitarbeiter für die Normen des humanitären Völkerrechts übernimmt das Internationale Komitee vom Roten Kreuz einen wichtigen Part. Die Organisation bemüht sich bereits seit längerer Zeit um einen konstruktiven Dialog mit Unternehmen, die in Konfliktregionen tätig sind. ${ }^{41}$ Ziel dieses Dialogs ist es, das humanitäre

41 International Committee of the Red Cross (ICRC), For the Private Sector: Humanitarian Responsibilities in War-prone Areas, <www.icrc.org/web/eng/ siteeng0.nsf/htmlall/private_sector_responsibility?OpenDocument>; Gilles Carbonnier/Marie-Servane Desjonquères, Corporate Responsibility - What Does It Mean for Humanitarian Action?, in: The Magazine of the International Red Cross and Red Crescent Movement, (2002) 3.
Verantwortungsbewusstsein dieser Akteure zu stärken und sie bei der Wahrnehmung ihrer Verantwortung zu unterstützen. ${ }^{42}$ In jedem Fall müsste sich bei solchen Unternehmen die Haltung durchsetzen, nur Firmen unter Vertrag zu nehmen, die sich zur Erfüllung bestimmter rechtlicher und ethischer Standards verpflichtet haben und ausreichend transparent agieren.

42 ICRC, The ICRC to Expand Contacts with Private Military and Security Companies, 4.8.2004, <www.icrc.org/web/eng/siteeng0.nsf/iwpList100>.

\title{
Möglichkeiten und Grenzen eines ständigen europä- ischen Sitzes im Sicherheitsrat der Vereinten Nationen
}

\author{
Jan Scheffler*
}

\begin{abstract}
For more than a decade, the reform of the United Nations' Security Council has been on the international agenda. In this context, wouldn't it be a good idea to give a permanent seat to the European Union in order to take into account the changing structure of international relations? Due to numerous obstacles which include the opposition of France and the United Kingdom as well as structural deficits of the CFSP, a European seat cannot be implemented in the medium term. But there are several leverages for improving the representation of common European interests in the Security Council. In this way, a »de facto-communitarization « of EU members' seats can be envisaged successively.
\end{abstract}

Keywords: Sicherheitsrat der Vereinten Nationen, Gemeinsame Außen- und Sicherheitspolitik (GASP), EU-Sitz, europäischer Außenminister

\section{Einleitung}

$\mathrm{D}$ er Millennium+5-Gipfel der Vereinten Nationen (VN) war für viele Betrachter eine Enttäuschung. ${ }^{1}$ Auch wenn einige Beschlüsse wie die grundsätzliche Verständigung über die Einrichtung eines Menschenrechtsrats herbeigeführt werden konnten, haben die Mitgliedstaaten in wesentlichen Punkten der ambitionierten Reformagenda von VN-Generalsekretär Kofi Annan keine Einigung erzielt. ${ }^{2}$ Dies gilt nicht zuletzt für die Neuordnung des Sicherheitsrats (SR), die nach heftigem Streit im Vorfeld des Gipfels bis auf weiteres verschoben ist.

Damit hat die Diskussion um die Reform des höchsten VNGremiums, dessen Struktur nach wie vor die Mächtekonstel-

\footnotetext{
* Doktorand an der Universität St. Gallen; M.A. in International Affairs and Governance (Universität St. Gallen), Diplômé de Sciences Po Paris, B.A. in Europäischen Studien (Universität Osnabrück).

1 Vgl. für eine kritische Bewertung die Analyse von Wagner (2005).

2 Vgl. das Abschlussdokument des Gipfels: Vereinte Nationen (2005).
}

lation vom Ende des Zweiten Weltkriegs widerspiegelt, aber keineswegs an Aktualität verloren. Die Zweifel an seiner Repräsentativität und Legitimität, an seiner Effizienz und Effektivität bestehen fort. Will der SR langfristig seiner in der VNCharta definierten Aufgabe nachkommen, die internationale Friedensordnung zu schützen, müssen seine Zusammensetzung und seine Arbeitsweise den weltpolitischen Bedingungen des 21. Jahrhunderts angepasst werden.

Über die jüngsten Scharmützel zwischen den Befürwortern unterschiedlicher Erweiterungsszenarien hinaus stellt sich die Frage nach einer stärkeren Einbindung der Europäischen Union (EU) in die Arbeit des SR. Wäre es nicht eine Option, im Zuge einer grundlegenden Reform einen ständigen Sitz $^{3}$ an

\footnotetext{
3 Im Zusammenhang mit einer EU-Repräsentanz im SR wird stets nur von einem ständigen Sitz gesprochen. Eine nicht-ständige Mitgliedschaft wäre wohl in jeder Beziehung dysfunktional und soll daher im Weiteren keine Beachtung finden.
} 
die EU zu vergeben? Den Implikationen eines solchen innovativen Konzeptes ist der folgende Beitrag gewidmet. Welche Überlegungen stehen hinter diesem Projekt? Welche Chancen bietet es, welche Risiken birgt es? Und welche konkreten institutionellen Arrangements sind denkbar?

Nun greift man der Analyse nicht vor, wenn man angesichts der festgefahrenen Reformdiskussion in den VN einerseits und der Krise um die Ratifikation des EU-Verfassungsvertrages andererseits zumindest die kurzfristige Schaffung eines gemeinsamen europäischen Sitzes im SR für unwahrscheinlich hält. ${ }^{4}$ Insofern erscheint es angemessen, auch mögliche Alternativen im Blick zu behalten, die mit den übergreifenden Zielen eines gemeinsamen europäischen Sitzes (Stärkung der EU als internationaler Akteur, Verbesserung von Repräsentativität und Handlungsfähigkeit des SR) im Einklang stehen.

\section{Die EU in den Sicherheitsrat: Argumente und Fürsprecher im Überblick}

\subsection{Die Ratio für eine permanente Mitgliedschaft der EU}

Die europäische Integrationsgemeinschaft hat sich seit jeher dem System der VN verbunden gefühlt. Im aktuell gültigen Vertrag über die Europäischen Union (EUV) ist in Art. 11 Abs. 1 als ein Ziel der Gemeinsamen Außen- und Sicherheitspolitik (GASP) »die Wahrung des Friedens und der internationalen Sicherheit entsprechend den Grundsätzen der Charta der Vereinten Nationen « definiert. Die EU und die VN haben einen sehr ähnlichen Blickwinkel auf die Welt, setzen sich für dieselben Werte und Ordnungsprinzipien (u.a. Demokratie, Rechtsstaatlichkeit, Marktwirtschaft, Nachhaltigkeit und Solidarität) ein und kooperieren in zunehmendem Maße auch im operativen Bereich wie im Falle der »Artemis«-Operation im Kongo oder des »Concordia«-Einsatzes in Mazedonien. ${ }^{5}$ Damit ist aber nicht bereits die Frage beantwortet, warum dieser offensichtlich fruchtbaren Kooperation noch besser als unter dem gegenwärtigen Regime gedient wäre, wenn die EU über einen eigenen permanenten Sitz im SR verfügte.

Dafür lassen sich aus dem Blickwinkel beider Organisationen Argumente anführen: Aus Sicht der VN wäre es sehr nützlich, einen starken und verlässlichen Akteur, der die gleichen Ziele und Grundsätze hat, am Tisch des SR zu wissen. Als regionaler Akteur, der nicht ausschließlich den nationalen Interessen der EU-Mitgliedstaaten verpflichtet wäre, könnte die EU mit einer eigenen Vertretung im höchsten Gremium der VN nachdrücklich für eine verantwortungsbewusste »global governance « eintreten.

Aus Sicht der EU wäre eine ständige Mitgliedschaft im SR insofern gewinnbringend, als diese dort direkt für ihre Ziele,

4 Wenn hier wie im Folgenden von einem »europäischen Sitz« gesprochen wird, ist damit immer eine gemeinsame Vertretung der EU-Mitgliedstaaten im SR gemeint, nicht aber eine kollektive Repräsentanz aller europäischen Staaten.

5 Vgl. Europäische Union (2004: 6).
Grundsätze und Interessen eintreten könnte. EU-Standpunkte haben erheblich mehr Chancen, in der internationalen Politik gehört zu werden, wenn sie (auch institutionell) einheitlich vorgebracht werden. Dafür bietet eine permanente Mitgliedschaft im SR - mit einem dort die EU vertretenden Außenminister oder Hohen Vertreter für die GASP - günstige Aussichten. Auch im Sinne der Repräsentativität nach innen würde ein gemeinsamer europäischer Sitz einen Fortschritt bedeuten, weil an dessen politisch-inhaltlicher Ausgestaltung alle EU-Mitgliedstaaten beteiligt wären.

Bei diesen Perspektiven darf aber nicht übersehen werden, dass die EU kein einheitlicher Block und schon gar kein Staat ist, der als SR-Mitglied staatsähnliche Handlungsfähigkeit besäße. Über die damit zusammenhängenden Probleme wird noch zu sprechen sein. Interessant ist aber, dass sich der Rohbauzustand der GASP auch als Argument für einen gemeinsamen Sitz anbringen lässt. In diesem Fall würde die institutionelle Integration quasi vorausgeschickt und den Zwang schaffen, dass sich die Realität der politischen Prozesse und Entscheidungsverfahren den Funktionserfordernissen der gemeinsamen Repräsentanz im SR anpasst.

\subsection{Politische Unterstützung und deren Motive}

Betrachtet man nun den Kreis derer, welche die vorstehenden Argumente teilen, ergibt sich ein bemerkenswertes Bild. Erstens handelt es sich bei den Befürwortern eines gemeinsamen EU-Sitzes nicht um ein paar versprengte Idealisten, auch wenn ihre Motive variieren; zweitens sind sie quer durch alle politischen Parteien anzutreffen; und drittens werden sie immer zahlreicher. Einige Beispiele: Der ehemalige italienische Ministerpräsident Giulio Andreotti hatte schon 1990 vor dem Europäischen Parlament gefordert, Frankreich und das Vereinigte Königreich sollten auf ihre beiden Sitze zugunsten von Japan und der EG verzichten. ${ }^{6}$ Zuletzt haben sich die italienischen Außenminister Frattini ${ }^{7}$ und Fini ${ }^{8}$ als Befürworter eines europäischen Sitzes hervorgetan.

Auch in Deutschland spielte in den vergangenen Jahren die europäische Dimension im SR immer wieder eine Rolle. Die inzwischen abgewählte rot-grüne Bundesregierung sprach in ihrem Koalitionsvertrag aus dem Jahr 1998 von einem »grundsätzlich bevorzugte[n] europäische[n] Sitz«. ${ }^{9}$ Bekanntlich ist sie allerdings von diesem Ziel zugunsten des Werbens um eine ständige deutsche Mitgliedschaft abgerückt. Dennoch sind die Stimmen, die sich für eine EU-Repräsentanz im SR aussprechen, keineswegs verklungen, wie die Ansichten des ehemaligen CDU-Vorsitzenden Schäuble, ${ }^{10}$ des EVP-Fraktionsvorsitzenden Pöttering ${ }^{11}$ oder des FDP-Chefs Westerwelle $\mathrm{e}^{12}$ belegen.

\footnotetext{
6 Vgl. Winkelmann (2000: 416)

7 Vgl. Frattini (2004).

8 Vgl. Fini (2004).

9 Sozialdemokratische Partei Deutschlands und BÜNDNIS 90/DIE GRÜNEN (1998).

10 Vgl. Schäuble (2005).

11 Vgl. Bacia (2004b).

12 Vgl. Carstens (2003).
} 
Wenig Beachtung in der Öffentlichkeit hat ganz zu Unrecht eine Entschließung des Europäischen Parlaments auf der Grundlage des Laschet-Berichts gefunden. ${ }^{13}$ Danach soll die EU als ständiges Mitglied in den SR aufgenommen werden, sobald sie Rechtspersönlichkeit besitzt. Ihrem integrationspolitischen Zeichen haben die Europaparlamentarier in ihrer Entschließung dank einer Auslassung sogar ein "Sahnehäubchen « aufgesetzt: Der Antrag, in den Text einen Passus aufzunehmen, wonach Frankreich und das Vereinigte Königreich ihre ständigen Sitze in jedem Fall behalten dürften, wurde abgelehnt. ${ }^{14}$

Ganz so weit möchten die Protagonisten der europäischen Außenpolitik nicht gehen. Aber auch »Mr. GASP« Javier Solana hat zumindest seine Sympathie für einen europäischen Sitz erklärt. ${ }^{15}$ Und EU-Außenkommissarin Benita Ferrero-Waldner hat sich wiederholt ${ }^{16}$ für die gemeinsame EU-Vertretung im SR ausgesprochen.

Gemeinsam ist nahezu allen Forderungen nach einem EUSitz, dass sie keine oder nur sehr vage Aussagen zu den angestrebten Modalitäten einer Implementierung machen. Dies deutet darauf hin, dass sich hinter der Parteinahme für die gemeinsame europäische Vertretung sehr unterschiedliche Motive verbergen können. Während sich auf der einen Seite die föderalistisch orientierten Kräfte von dem kontinuierlichen Verweis auf das (noch) schemenhafte Ziel der gemeinsamen Vertretung eine Stärkung der GASP versprechen, kann die gleiche Forderung auf der anderen Seite eine Feigenblattfunktion erfüllen. Es ist recht gefahrlos, sich als »guter Europäer « darzustellen, obwohl eigentlich andere Absichten verfolgt werden, da kurzfristig mit einer Umsetzung des Projekts "gemeinsamer Sitz« ohnehin nicht zu rechnen ist. Die italienische Position unterliegt zumindest teilweise dem Verdacht, dieser Logik zu folgen. ${ }^{17}$

\section{Hindernisse auf dem Weg zu einem ständigen EU-Sitz}

\subsection{Die EU und die alten ständigen Mitglieder Frankreich und Großbritannien}

Alle anderen Schwierigkeiten bei dem Bemühen um einen gemeinsamen EU-Sitz werden davon überlagert, dass Frankreich und Großbritannien wenig bis keine Bereitschaft zeigen, ihre Privilegien als ständige SR-Mitglieder und Vetomächte zugunsten einer gemeinsamen europäischen Repräsentanz aufzugeben. Beide Länder haben ihre Sitze im SR immer als nationale Vertretung verstanden - und nicht etwa als europäische, auch nicht seit der Einführung von Konsultationsmechanismen mit dem Vertrag von Maastricht.

13 Vgl. Europäisches Parlament (2003).

14 Vgl. dazu Coûteaux (2004). In einer Entschließung zur VN-Reform vom 09.06.2005 haben die Straßburger Parlamentarier ihre Position im Wesentlichen bestätigt, sprechen nun allerdings etwas moderater davon, »einen zusätzlichen ständigen Sitz an die Europäische Union zu vergeben«. Vgl. Europäisches Parlament (2005).

15 Vgl. Mann (1999).

16 Vgl. etwa Ferrero-Waldner (2004) und Berliner Zeitung (2005).

17 Siehe dazu Abschnitt 3.3.
Wäre es daher vielleicht denkbar, einen EU-Sitz zusätzlich zu den Vertretungen Frankreichs und Großbritanniens oder womöglich in Ergänzung zu allen bisherigen Sitzen von EU-Mitgliedstaaten zu schaffen? Da der EU-Sitz dazu dienen soll, dass Europa mit einer Stimme spricht, wird eine solche Konstruktion zu Recht als »unlogisch und in sich widersprüchlich ${ }^{18}$ bezeichnet. Dieser erste Eindruck verfestigt sich, wenn man die rechtlichen Rahmenbedingungen für eine EU-Mitgliedschaft im SR betrachtet.

\subsection{Die Mitgliedschaft der EU im Sicherheitsrat als völkerrechtliches Problem}

Die zentrale völkerrechtliche Barriere für eine Mitgliedschaft der EU im SR liegt in Art. 4 Abs. 1 VN-Charta begründet: Mitglieder der Weltorganisation können nur Staaten werden. Die EU aber kann beim besten Willen nicht als Staat gelten. Daher würde es für einen EU-Sitz im SR nicht genügen, die Bestimmungen des Art. $23 \mathrm{VN}$-Charta bezüglich dessen Zusammensetzung zu ändern. Da dort von »Mitgliedern der Vereinten Nationen «, aus denen der SR besteht, die Rede ist, und da Art. 4 Abs. 1 VN-Charta wie erwähnt für eine solche Mitgliedschaft die Voraussetzungen benennt (nämlich u.a. die Staatseigenschaft), kann die EU nicht gleichsam durch die Hintertür in den SR aufgenommen werden.

Selbstverständlich könnte man die VN-Charta dahin gehend ändern, dass die Mitgliedschaft nicht mehr nur Staaten offen stünde. Allerdings ist zu bezweifeln, dass dafür die Formierung der notwendigen Mehrheit gemäß des Vertragsänderungsverfahrens nach Art. 108 VN-Charta inklusive Vetorecht der ständigen Mitglieder gelänge. Auch die konkrete Umformulierung der Charta würde sich sehr problematisch gestalten: Entweder man würde durch eine explizite »lex EU« eine ungerechtfertigte Sonderbehandlung festschreiben oder aber durch einen allgemeinen Bezug zu regionalen Organisationen die unlösbare Frage aufwerfen, welche der unzähligen regionalen Organisationen noch Mitglied der VN oder gar des SR werden könnten. ${ }^{19}$ Zudem müssten der Leitsatz der souveränen Gleichheit aller Mitglieder (Art. 2 Ziff. 1 VN-Charta) und das Problem der Mitgliedschaft von Staaten und bzw. oder regionalen Organisationen in der Generalversammlung dann überdacht werden.

Die Vollmitgliedschaft internationaler Organisationen in anderen internationalen Organisationen ist im Völkerrecht zwar kein unbekannter Fall. Die wenigen vorliegenden Fälle - wie die Mitgliedschaft der EG in der WTO und in der FAO - eignen sich allerdings nur sehr begrenzt als Vorbild für die $\mathrm{VN}$ und den SR. In der FAO ist die EG seit einer Charta-Änderung im Jahr 1991 Vollmitglied. Die Staaten der EG sind neben der Gemeinschaft aber weiterhin als eigenständige Mitglieder in der Welternährungsorganisation vertreten. Dieses schwierige Nebeneinander wird institutionell durch die Formel der alternativen Ausübung der Mitgliedschaftsrechte je nach Politikfeld und Zuständigkeitsbereich geregelt.

18 Bacia (2004a).

19 Vgl. Govaere, Capiau \& Vermeersch (2004: 177). 
Wendet man dieses Modell auf den VN-SR an, so ergeben sich diverse Schwierigkeiten. Die erste betrifft die Kompetenzfrage: Exklusive Zuständigkeiten der EU bestehen im Bereich der Außen- und Sicherheitspolitik bisher nicht. Zweitens hat die EU im Gegensatz zur EG (noch) keine explizite eigene Rechtspersönlichkeit. Drittens ist es nur schwer vorstellbar, wie der SR als hoch politisches und hoch sensibles Organ seine Funktionsfähigkeit aufrechterhalten könnte, wenn es zu einer alternativen Ausübung der Mitgliedschaftsrechte kommen sollte. Insofern erscheint die radikalere Lösung eines originären EU-Sitzes im SR - die EU-Mitgliedstaaten müssten also auf eine eigene nationale Repräsentanz verzichten - als weniger heikel. Im Bereich der internationalen Fischereiorganisationen gibt es Beispiele für eine solche Lösung. ${ }^{20}$ Der entscheidende Unterschied zur Außen- und Sicherheitspolitik und damit zum VN-SR bleibt aber, dass die EG mit der Gemeinsamen Fischereipolitik über eine exklusive Zuständigkeit verfügt, während die GASP in der EU im intergouvernementalen Rahmen verortet ist.

\subsection{Der Streit um Deutschlands Wunsch nach einer ständigen Mitgliedschaft}

Vor dem Hintergrund der Debatte um eine gemeinsame EUVertretung im SR ist das Streben Deutschlands nach einem eigenen ständigen Sitz besonders interessant: Ebnet ein solcher den Weg für eine genuin europäische Komponente im SR, oder bedeutet er den Rückfall in überholte nationalstaatliche Muster der Außenpolitik?

Die beiden Vetomächte Frankreich und Großbritannien haben nach anfänglichem Widerstand den deutschen Wunsch nach einem ständigen Sitz unterstützt. ${ }^{21}$ Dies geht aber mitnichten mit einem begeisterten Eintreten für eine Vergemeinschaftung der europäischen SR-Sitze einher. Zumindest unterschwellig dürfte in der französischen und britischen Position die Hoffnung mitschwingen, dass ein ständiger deutscher Sitz die in ihren Augen lästige Diskussion um die gemeinsame europäische Vertretung endgültig verstummen ließe.

Ist - europäisch gedacht - ein ständiger deutscher Sitz schon aus diesem Grund abzulehnen? Nicht unbedingt. Für die langfristige Perspektive einer europäischen Vertretung wäre es nicht nachteilig, wenn der SR bereits jetzt eine grundsätzliche Reformfähigkeit bewiese. Deutschland könnte in diesem Fall als ständiges SR-Mitglied durch eine nachhaltig integrativ ausgeprägte Außen- und Sicherheitspolitik einen wichtigen Beitrag zu einer stärkeren Europäisierung der VN-Politik der EU-Mitgliedstaaten leisten.

Das ist nicht widersprüchlich, wie einige Autoren meinen, ${ }^{22}$ die in der Regel die Möglichkeiten einer dynamischen Evolution verkennen; es ist aber auch kein Automatismus, der ganz von allein auf das europäische »Gute« zusteuert. Das fragile europäische (und internationale) Gleichgewicht scheint die Bundesregierung unter Gerhard Schröder bei ihrem aktiven, mitunter forschen Werben um einen deutschen Sitz allerdings

20 Vgl. ausführlich Frid (1995: 319ff).

21 Vgl. Andreae (2002: 200ff).

22 Vgl. etwa Stelzenmüller (2004) und Zumach (2005). nicht immer im Blick gehabt zu haben. So hat der Streit mit Italien, das sich vehement gegen eine Erweiterung des SR um neue ständige Mitglieder wehrt, dem koordinierten Auftreten der EU zweifellos geschadet. Während Berlin die Partner also nicht genügend eingebunden hat, muss sich Rom seinerseits vorhalten lassen, eine prinzipielle Blockadehaltung eingenommen zu haben, die das erklärte Ziel mehrerer italienischer Regierungen seit den 1990er Jahren - nämlich die Schaffung eines EU-Sitzes - gerade nicht fördert. ${ }^{23}$

Ob dieser Antagonismus in der italienischen Politik auf eine inkonsistente Strategie zurückzuführen ist oder doch auf die rhetorische Instrumentalisierung des EU-Sitzes als »Feigenblatt«, um eine national motivierte Gangart gegen die deutschen Ambitionen zu verbrämen, ist schwer zu sagen. In jedem Fall kann als Ergebnis festgehalten werden, dass die Option eines ständigen deutschen Sitzes zwar zu einer Stärkung der europäischen Dimension im SR führen kann, aber nur dann, wenn sich alle beteiligten Akteure auf die Tugenden der Einbindung und der Konzertation besinnen.

\subsection{Strukturelle Defizite der Gemeinsamen Außen- und Sicherheitspolitik}

Die bisher genannten Probleme hängen alle zumindest indirekt mit der Struktur der GASP zusammen. Da sie intergouvernemental ausgelegt ist und nach dem Prinzip der Einstimmigkeit funktioniert, bietet sie praktisch keinen Hebel für eine gemeinsame Außenvertretung, falls sich die Regierungen der Mitgliedstaaten nicht einigen können oder wollen. Selbst wenn eine einheitliche Position vorliegt, beeinträchtigt der fehlende institutionelle Überbau die Effektivität der GASP.

Für die Artikulation europäischer Interessen im SR findet sich in Art. 19 Abs. 2 EUV eine komplizierte Regelung. Dort heißt es zunächst, dass sich die EU-Mitgliedstaaten, die auch im SR vertreten sind, untereinander abstimmen werden und die übrigen Mitgliedstaaten in vollem Umfang unterrichten. Dem Wortlaut ist also zu entnehmen, dass die gegenseitige Abstimmung nicht unter allen EU-Mitgliedstaaten erfolgen muss, sondern nur unter denjenigen, die auch dem SR angehören. Zudem genießen Frankreich und Großbritannien eine Sonderrolle: Als EU-Mitgliedstaaten, die ständige Mitglieder des SR sind, haben sie sich bei der Wahrnehmung ihrer Aufgaben für die Standpunkte und Interessen der Union einzusetzen, allerdings »unbeschadet ihrer Verantwortlichkeiten aufgrund der Charta der Vereinten Nationen«. Diese »Unbeschadet-Klausel« des Art. 19 Abs. 2 EUV eröffnet Frankreich und Großbritannien eine erhebliche Bewegungsfreiheit, die ihnen durchaus gelegen kommt. ${ }^{24}$

Die dem SR angehörenden europäischen Staaten nehmen ihre Aufgabe in dem Gremium also grundsätzlich individuell wahr; sie handeln nicht als EU. ${ }^{25}$ Zwar finden bisweilen abgestimmte EU-Positionen explizit Eingang in die Diskussionen im SR. Sie

23 Zusammenfassend zu den Motiven des italienischen Handelns sowie zur Entwicklung der italienischen Position vgl. Andreae (2002: 250ff).

24 Vgl. Regelsberger \& Kugelmann (2003: Art. 19, Rn 2).

25 Vgl. Kaufmann-Bühler (2003: Art. 11, Rn 26). 
werden von der Ratspräsidentschaft in offenen SR-Sitzungen präsentiert oder - in seltenen Fällen - auch von »Mr. GASP« Javier Solana, wenn er vom SR dazu eingeladen wird. Das ändert aber nichts daran, dass der momentane Zustand der GASP einem ständigen EU-Sitz entgegensteht. Das Fehlen einer supranationalen Entscheidungsstruktur verhindert, dass eine solche europäische Vertretung effektiv funktionieren würde. Unter dem Einstimmigkeitsprinzip wäre die EU bei divergierenden Interessen ihrer Mitglieder mangels eindeutiger Weisungen folglich gezwungen, sich im SR zu enthalten. Damit wäre Europa handlungsunfähig und könnte seine Verantwortung auf der Weltbühne nicht wirkungsvoll wahrnehmen. ${ }^{26}$

All dies soll aber nicht nahe legen, dass eine stärkere Akzentuierung der europäischen Komponente im SR unmöglich ist. Vielversprechender als institutionell mit dem Kopf durch die Wand zu gehen, scheint es dabei allerdings zu sein, die bestehenden GASP-Instrumente besser auszunutzen und behutsam weiterzuentwickeln.

\section{Jenseits eines ständigen Sitzes: Ansatzpunkte für eine wirksame Vertretung gemeinsamer europäischer Interessen}

\subsection{Effektive Nutzung der GASP-Instrumente}

Die Europäische Sicherheitsstrategie, die im Dezember 2003 von der EU verabschiedet wurde, enthält eine grundlegende Feststellung: »Entscheidend (...) ist, dass wir stärker sind, wenn wir gemeinsam handeln. ${ }^{27}$ Wie lässt sich diese Einsicht für ein integrativeres europäisches Auftreten im SR nutzen? Der Schlüssel zu einer besseren Aufgabenerfüllung liegt vor allem in der Korrektur gewisser eingefahrener Verhaltensmuster. Dies betrifft in erster Linie die Unterrichtungskultur zwischen europäischen Mitgliedern und Nicht-Mitgliedern des SR. Das Ziel sollte es sein, eine wirkliche Koordinierung zu gewährleisten, bei der die EU-Mitglieder im SR von ihren europäischen Partnern eindeutige Informationen über die gemeinsame Position zu sicherheitspolitisch relevanten Fragen bekommen und diese dann auch entsprechend einbringen. Unterstützend könnte in diesem Kontext ein stärkerer Einbezug der betreffenden Ratsarbeitsgruppe in Brüssel (CONUN) oder auch des Politischen und Sicherheitspolitischen Komitees (PSK) wirken. ${ }^{28} \mathrm{Zu}$ bedenken wäre ferner die Inkorporierung von Vertretern der nicht dem SR angehörenden EU-Mitgliedstaaten und von Repräsentanten der Gemeinschaftsinstitutionen in die Delegationen von europäischen SR-Mitgliedern. ${ }^{29}$

Diese Vorschläge bringen keine spektakulären Neuerungen, sondern beschreiben vielmehr einen »langen mühsamen Marsch durch die Ebene ${ }^{30}$ der auf eine schrittweise faktische »Vergemeinschaftung « der von den EU-Staaten eingenom-

26 Vgl. Winkelmann (2000: 436)

27 Vgl. Europäische Union (2003: 14)

28 Vgl. Sucharipa (2003: 790), Europäische Kommission (2003: 17f).

29 Skeptisch dazu: Kaufmann-Bühler (2003: Art. 19, Rn 12).

30 Sucharipa (2003: 794). menen Sitze im SR abstellt. Dafür bedarf es zunächst gar keiner Änderung des EUV, sondern lediglich einer gemeinschaftsfreundlicheren, offensiveren Auslegung des Art. 19 Abs. 2.

Mit Blick auf die EU-internen Entscheidungsverfahren wäre es bereits ein Fortschritt, wenn die im Vertrag vorgesehenen Möglichkeiten zu Mehrheitsentscheidungen und vor allem zur konstruktiven Enthaltung tatsächlich genutzt würden. Denkbar - und institutionell mutiger - wäre es natürlich, Entscheidungen per qualifizierter Mehrheit auch im GASP-Rahmen als Grundregel einzuführen und gegebenenfalls gewisse Ausnahmen zuzulassen. Die Erfahrungen mit dem Regime der Mehrheitsentscheidungen in der EG-Säule haben gezeigt, dass allein die Möglichkeit einer veritablen Abstimmung eine erfolgreiche Konsenssuche fördert, ohne dass es dann letztlich zum Votum kommt. ${ }^{31}$

\subsection{Perspektiven im Verfassungsvertrag}

Angesichts der aufgeworfenen Probleme ist zu fragen, ob der Vertrag über eine Verfassung für Europa (VVE) Neuerungen bereithält, welche jenen abhelfen könnten. Selbst wenn der VVE nicht wie ursprünglich geplant in Kraft tritt, besitzen die dort festgeschriebenen Regelungen zur GASP Chancen, später in der einen oder anderen Form doch noch umgesetzt zu werden, weil der Streit um die Ratifikation diese Bestimmungen kaum betrifft.

Im VVE ist das auswärtige Handeln der Union erstmals in einem einheitlichen Titel zusammengefasst worden; die Säulenstruktur existiert nicht mehr. Die grundlegende intergouvernementale Logik der GASP und damit die Dominanz der Mitgliedstaaten bleiben aber bestehen. Des Weiteren sucht man eine eindeutige Regelung zum Beitritt der EU zu internationalen Organisationen und erst recht einen Verweis auf einen anzustrebenden Sicherheitsratssitz vergeblich. ${ }^{32}$ Insgesamt ist aber trotz restriktiver Beschlussfassungsmodi eine gewisse Verbesserung der außenpolitischen Handlungsfähigkeit der Union zu erwarten ${ }^{33}$ - unter anderem aufgrund der Verleihung einer eigenen Rechtspersönlichkeit an die EU sowie der Einführung eines hauptamtlichen Präsidenten des Europäischen Rates, eines Europäischen Auswärtigen Dienstes und eines Außenministers der Union.

Bezüglich der Neufassung des Art. 19 Abs. 2 EUV hat man die umstrittene »Unbeschadet-Klausel« zwar übernommen, den Bezug zu den ständigen Mitgliedern aber gestrichen. Damit entfällt für das Vereinigte Königreich und Frankreich die derzeit noch festgeschriebene Exklusivität des »UnbeschadetVorrechts«. Es gilt nun genauso für die anderen EU-Mitgliedstaaten mit Sitz im SR und ist nicht als Privileg, sondern ausschließlich als gemeinschaftsrechtliche Bezugnahme auf die VN-Charta zu werten.

Diese nur vorsichtigen Weiterentwicklungen bieten noch keine Gewähr für eine stärker europäisch ausgelegte Aufgabenwahrnehmung der im SR vertretenen EU-Mitgliedstaaten. Im

31 Vgl. Jopp, Reckmann \& Regelsberger (2002: 232).

32 Vgl. Govaere et al. (2004: 186).

33 Vgl. Jopp \& Regelsberger (2003: 562). 
Kontext einer fortschreitenden Vergemeinschaftung bedeuten sie aber ein leichtes Plus, weil sie eine zunehmende Bewusstseinsbildung für das Erfordernis einer starken und handlungsfähigen Außenvertretung erkennen lassen.

\subsection{Aufwertung des europäischen Außenmini- sters bzw. des Hohen Vertreters}

Der Wille der EU, nach außen mit einer Stimme zu sprechen, findet im VVE besonders in der Schaffung des Amtes eines europäischen Außenministers Ausdruck. In einer »Doppelhut«-Lösung zugleich Beauftragter des Rates für die GASP und Vizepräsident der Europäischen Kommission, wird der Außenminister u.a. für die Leitung der GASP sowie für die Organisation der Koordinierung mitgliedstaatlichen Handelns in internationalen Organisationen und auf internationalen Konferenzen zuständig sein. Dem Außenminister sind zwar im Prinzip die Hände gebunden, wenn die Mitgliedstaaten nicht zu einem Konsens kommen, und er könnte in seiner Mehrfachfunktion auch in einen Loyalitätskonflikt geraten. Er verfügt aber über weit reichende Möglichkeiten, selbst in den Politikformulierungs- und Entscheidungsprozess einzugreifen. ${ }^{34}$

Mit konkretem Bezug auf den SR verfügt der VVE, dass, wenn die Union einen Standpunkt zu einem im SR zu behandelnden Thema festgelegt hat, der Außenminister diesen vertreten soll. Selbstverständlich greift diese Formel nur, falls ein gemeinsamer Standpunkt überhaupt existiert. Im Grundsatz aber wäre die Systematisierung der Präsentation von EU-Positionen der Visibilität der GASP in jedem Fall zuträglich.

Eine solche Reform wäre auch dann anzustreben, wenn der VVE vorläufig nicht in Kraft träte. Unterhalb der förmlichen Vertragsänderung haben die EU-Mitgliedstaaten die Möglichkeit, häufiger und klarer als bisher den Hohen Vertreter für die GASP als Sprachrohr und Verhandlungspartner im SR zu mandatieren. Zweifelsohne sind die Kompetenzen des Hohen Vertreters gegenwärtig wesentlich beschränkter, als es diejenigen eines zukünftigen Außenministers wären. Doch der bisherige Amtsinhaber Javier Solana hat bewiesen, dass es möglich ist, der Rolle des Hohen Vertreters auch mit begrenzten institutionellen Voraussetzungen eine eigene Kontur zu geben. Mit der Unterzeichnung des VVE haben die EU-Mitgliedstaaten deutlich gemacht, dass sie prinzipiell ein stärker gemeinschaftlich ausgerichtetes Handeln der EU im SR wünschen. Darauf könnte der Hohe Vertreter für die GASP aufbauen, selbst wenn er vorläufig weiter unter den Bedingungen des Vertrages von Nizza agieren muss.

\section{Schlussbetrachtung}

Die Untersuchung hat gezeigt, dass die Schaffung einer gemeinsamen europäischen Vertretung im SR auf mittlere Sicht nicht realisierbar ist. Weder auf der internationalen noch auf der europäischen Ebene sind die Hindernisse, die einem

34 Vgl. im Detail Jopp \& Regelsberger (2003: 558). ständigen EU-Sitz entgegenstehen, mit einfachen Mitteln zu überwinden. Gewiss bedeuten die gescheiterten Verfassungsreferenden in Frankreich und den Niederlanden ebenfalls einen Rückschlag für eine schnelle Modernisierung der GASP. Auf diese Entwicklung kann Resignation aber keine politisch sinnvolle Reaktion sein. Die EU wird als internationaler Akteur im sicherheitspolitischen Bereich benötigt, und es gibt zahlreiche Möglichkeiten, mit denen sie auch im SR ihr Profil schärfen kann. Der effektive Gebrauch der Instrumente, welche die GASP anbietet, würde diesbezüglich wertvolle Dienste leisten.

Wenn die EU und namentlich ihre Mitgliedstaaten die dargelegten Potenziale mittelfristig ausschöpfen, werden sie dadurch auf dem Weg zu einer substanziellen Vergemeinschaftung ihrer sicherheitsratsrelevanten Politiken weit vorangeschritten sein. Möglicherweise stellt sich die Frage nach einer Formalisierung durch eine kollektive Vertretung im höchsten Gremium der VN dann neu.

\section{Literaturverzeichnis}

Andreae, L. (2002). Reform in der Warteschleife. Ein deutscher Sitz im UN-Sicherheitsrat? München: Oldenbourg.

Bacia, H. (2004a). Außenpolitische Vielstimmigkeit. Die Bewerbung Berlins um einen ständigen Sitz im UN-Sicherheitsrat passt ins europäische Bild. Frankfurter Allgemeine Zeitung (01.10.2004).

Bacia, H. (2004b). Pöttering für ständigen EU-Sitz im Sicherheitsrat. Frankfurter Allgemeine Zeitung (26.08.2004).

Berliner Zeitung (2005). »Unsere Lebensqualität als Exportartikel« - EU-Kommissarin Ferrero-Waldner über die Beziehung zu China und Sitze im UN-Sicherheitsrat (02.08.2005).

Carstens, P. (2003). FDP - EU in den Sicherheitsrat. Frankfurter Allgemeine Zeitung (08.04.2003).

Coûteaux, P.M. (2004). La France, l'Europe et l'Onu - Tribune Libre. »La preuve«. Gefunden am 03.11.2005 unter http://www.voxlatina.com/vox_dsp2.php3?art=1786.

Europäische Kommission (2003). The European Union and the United Nations: the choice of multilateralism. Communication from the Commission to the Council and the European Parliament (COM(2003) 526 final).

Europäische Union (2003). Ein sicheres Europa in einer besseren Welt. Europäische Sicherheitsstrategie. Brüssel: Europäische Union.

Europäische Union (2004). The Enlarging European Union at the United Nations: Making Multilateralism Matter. Brüssel: Europäische Union.

Europäisches Parlament (2003). Bericht über Beziehungen zwischen der Europäischen Union und den Vereinten Nationen (»Laschet-Bericht«) (A5-0480/2003). 
Europäisches Parlament (2005). Entschließung zur Reform der Vereinten Nationen vom 09.06.2005 (P6_ TA(2005)0237).

Ferrero-Waldner, B. (2004). The Future of the UN: Results of the Kofi Annan High Level Panel on Threats, Challenges, and Change. Conference organized by the European Policy Centre and the Konrad-Adenauer-Stiftung. Brüssel (08.12.2004). Gefunden am 03.11.2005 unter http://www.europa-eu-un.org/articles/de/article_4136_ de.htm.

Fini, G. (2004). No additional permanent seats. International Herald Tribune (07.12.2004).

Frattini, F. (2004). UN-Sicherheitsrat: In der Einbahnstraße nationaler Egoismen. Frankfurter Allgemeine Zeitung (27.09.2004).

Frid, R. (1995). The Relations Between the EC and International Organizations. Legal Theory and Practice. Amsterdam: Universiteit van Amsterdam.

Govaere, I., Capiau, J. \& Vermeersch, A. (2004). In between-seats: The Participation of the European Union in International Organizations. European Foreign Affairs Review, 9, 155-187.

Jopp, M. \& Regelsberger, E. (2003). GASP und ESVP im Verfassungsvertrag - eine neue Angebotsvielfalt mit Chancen und Mängeln. Integration, 4, 550-563.

Jopp, M., Reckmann, J. \& Regelsberger, E. (2002). Ansatzpunkte und Optionen zur institutionellen Weiterentwicklung von GASP und ESVP. Integration, 3, 230-237.

Kaufmann-Bühler, W. (2003). Kommentar zu den Artikeln 11, 12, 16 und 19 EUV. In E. Grabitz \& M. Hilf (Hrsg.), Das Recht der Europäischen Union, Kommentar (Loseblattsammlung). München: C.H. Beck.

Mann, M. (1999). Solana Backs Fixed EU Seat on the Security Council. Reuters, 17.11.1999. Gefunden am 03.11.2005 unter http://www.globalpolicy.org/security/reform/solana.htm.
Regelsberger, E. \& Kugelmann, D. (2003). Kommentar zu den Artikeln 11, 19 und 23 EUV. In R. Streinz (Hrsg.), EUV/ EGV. Vertrag über die Europäische Union und Vertrag zur Gründung der Europäischen Gemeinschaft. München: C.H. Beck.

Schäuble, W. (2005). Ständiger deutscher Sitz im Sicherheitsrat? Nutzen weder für Deutschland noch für die Uno. Neue Zürcher Zeitung (05.02.2005).

Sozialdemokratische Partei Deutschlands und BÜNDNIS 90/DIE GRÜNEN (1998). Aufbruch und Erneuerung - Deutschlands Weg ins 21. Jahrhundert, Koalitionsvereinbarung. Gefunden am 03.11.2005 unter http://www.datenschutz-berlin.de/doc/de/koalo/.

Stelzenmüller, C. (2004). Wie einen Kropf. So dringend braucht die Welt einen ständigen deutschen Sitz im UN-Sicherheitsrat. Die Zeit (22.07.2004).

Sucharipa, E. (2003). Die Gemeinsame Außen- und Sicherheitspolitik (GASP) der Europäischen Union im Rahmen der Vereinten Nationen. In J.A. Frowein et al. (Hrsg.), Verhandeln für den Frieden: liber amicorum Tono Eitel (S. 773-796). Berlin: Springer.

Vereinte Nationen (2005). 2005 World Summit Outcome. General Assembly A/60/L.1. New York: Vereinte Nationen.

Wagner, B. (2005). Verpasste Gelegenheit. Vor allem die USA haben den UN-Reformgipfel torpediert. Internationale Politik, 10, 76-83.

Winkelmann, I. (2000). Europäische und mitgliedstaatliche Interessenvertretung in den Vereinten Nationen. Zeitschrift für ausländisches öffentliches Recht und Völkerrecht, Band 60, 413-445.

Zumach, A. (2005). Überflüssig wie ein Kropf. Zur Frage eines deutschen Ständigen Sitzes im Sicherheitsrat. Vereinte Nationen, 1, 7-8. 